\title{
Morphology Effect on Geometry of Photoinduced Charge-Separated State in P3HT:PCBM Blend Films as Studied by Time-Resolved EPR Spectroscopy
}

\author{
Taku Miura, Takashi Tachikawa, and Yasuhiro Kobori* \\ Department of Chemistry, Graduate School of Science, Kobe University, \\ 1-1 Rokkodai-cho, Nada-ku, Kobe 657-8501, Japan
}

Keywords: organic photovoltaic, poly(3-hexylthiophene, charge-separation, geometry, photoactive layer

\section{Introduction}

The organic photovoltaic (OPV) [1] devises have recently attracted great attention as the next generation thin-film solar cells that can be low-cost, flexible and light. The spin coating method from mixed solutions consisting of conjugate polymers as the electron donors (D) and fullerene derivatives as the acceptors (A) has been employed to produce the solid photoactive layer of the OPV cells [1]. Recent progresses in the organic materials [2] and in device structures [3] have enabled the efficient OPV cells as high as $12 \%$ in the light energy conversion efficiency. In the organic photoactive layers, it is well known that the molecules are self-organized by the polymers and by the fullerene derivatives, respectively to generate the bulk heterojunction (BHJ) [4] interfaces that are induced by the phase segregations. Blend films composed of the conjugated polymer of regioregular poly(3-hexylthiophene) (RR-P3HT) and the fullerene derivative of $[6,6]$-phenyl- $\mathrm{C}_{61}$-butyric acid ester $\left(\mathrm{PC}_{60} \mathrm{BM}\right)$ have attracted attention as a model system for the study of photoactive bulkheterojunction layer in the organic thin-film solar cells. However, only a few studies have directly characterized the geometries of photoinduced charge-separated (CS) state at the domain interface of the blend films $[5,6]$. Consequently, initial photoelectric conversion mechanisms have not been fully understood. In this study, we have directly observed the photoinduced CS state for the regioregular (RR)-P3HT:PC ${ }_{60} \mathrm{BM}$ blend films fabricated by the spin-coating method using time-resolved EPR
(TREPR) method to characterize the morphology effect on the orientation structure, the electronic property and the charge-dissociation dynamics following the primary CS process.

\section{Experimental}

The mixed solutions of the RR-P3HT: $\mathrm{PC}_{60} \mathrm{BM}$ (weight ratio $=2: 8,5: 5$ and 8:2) were prepared by dissolving them in 1,2-dichlorobenzene (ODCB). Blend films were fabricated by the spin-coating method on the cleaned cover-glasses at 3,000 rpm for $30 \mathrm{~s}$. Morphologies of the thin films were checked by the optical absorption bands of the RR-P3HT domains using the UV-vis spectrometer. The films in EPR quartz tubes were deaerated using a vacuum line to remove ODCB and oxygen. To conduct heat during the measurements and to avoid film exfoliation damages by the laser, 8:2 grycerol/water (v:v) mixture were added to the EPR tubes. These samples were deoxygenated again using the vacuum line. The X-band TREPR measurements were carried out using a Bruker EMX system at $T$ $=77 \mathrm{~K}$. Light excitations were performed by the second harmonics $(532 \mathrm{~nm})$ of a Nd:YAG laser (Continuum, Minilite II, fwhm $\sim 5$ ns) with a laser depolarizer.

\section{Results and discussion}

The UV-vis absorption spectrum of the 2:8 blend film showed a maximum band around 450 $\mathrm{nm}$, which is characteristic absorption of monomer P3HT in liquid solutions. In contrast, the $8: 2$ blend films showed the intense vibronic bands around 500-610 $\mathrm{nm}$ originating from the 


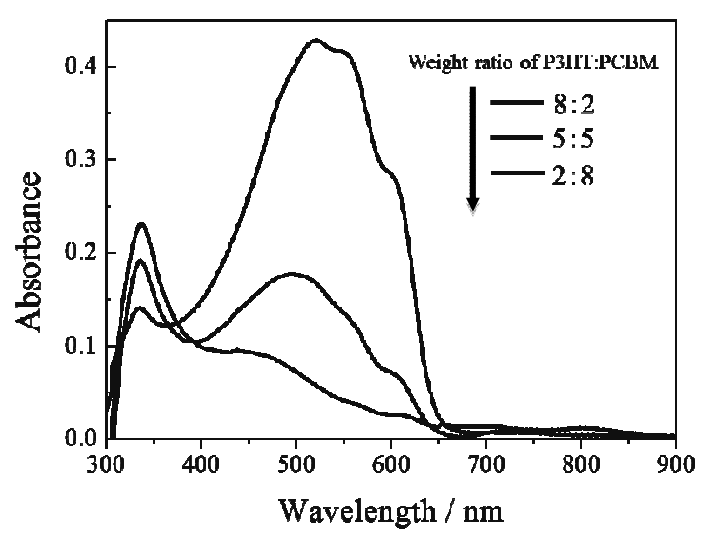

Fig. 1. UV-vis absorption spectra of the as-spun blend films with different weight ratios of RR-P3HT:PC ${ }_{60} \mathrm{BM}=8: 2$ (top at $500 \mathrm{~nm}$ ), 5:5 (middle) and 2:8 (bottom) fabricated from the ODCB solutions.

crystalline phase by the self-organized polymer molecules. The above results denote that the self-organization is disrupted when $\mathrm{P} 3 \mathrm{HT}$ content is small. The $340 \mathrm{~nm}$ band is assigned to $\mathrm{PC}_{60} \mathrm{BM}$. Fig. 2 shows TREPR spectra of the blend films of RR-P3HT:PC ${ }_{60} \mathrm{BM}=2: 8$ measured at $77 \mathrm{~K}$. The positive signal denoted by ' $A$ ' is the TREPR intensity (transverse magnetization) by the microwave absorption while the negative direction of ' $\mathrm{E}$ ' is the microwave emission. One can see that the broad $\mathrm{A} / \mathrm{A} / \mathrm{E} / \mathrm{E}$ polarization spectra are dominant as indicated by $\mathrm{A}$ and $\mathrm{E}$ for $0.5 \mu \mathrm{s}$ in the left of Fig. 2. Around the center field regions $(338 \mathrm{mT})$, however, additional spectrum components are superimposed. The broad $\mathrm{A} / \mathrm{A} / \mathrm{E} / \mathrm{E}$ component is assigned to a fine structure of the excited triplet state of ${ }^{3} \mathrm{PC}_{60} \mathrm{BM}^{*}$ generated in the isolated $\mathrm{PC}_{60} \mathrm{BM}$-rich region by the $\mathrm{S}_{1}-\mathrm{T}_{1}$ intersystem crossing of $\mathrm{PC}_{60} \mathrm{BM}$ [7]. In order to obtain the spectrum components at the center-field positions, we have performed the baseline corrections by the spectrum shapes of the ${ }^{3} \mathrm{PC}_{60} \mathrm{BM}^{*}$ around the center field regions as shown in the right of Fig. 2. The spectra in the right well agree with reported TREPR spectra obtained for the regiorandom $\mathrm{P} 3 \mathrm{HT}: \mathrm{PC}_{60} \mathrm{BM}$ drop-cast film [6] at $77 \mathrm{~K}$ and are explained by the sums of the broad $\mathrm{A} / \mathrm{E} / \mathrm{A} / \mathrm{E}$ components (as marked by $\mathrm{A}$ and $\mathrm{E}$ at the $0.6 \mu \mathrm{s}$ spectrum) and the sharp A/E/A signals. These signals have been explained by the spin correlated radical pair (SCRP) model using the g-factors of the P3HT polaron and of the PCBM anion radical [6], denoting that the photoinduced CS states are generated via the excited singlet P3HT. The coexisting of the initial broad $\mathrm{A} / \mathrm{E} / \mathrm{A} / \mathrm{E}$ components and the quite narrow $\mathrm{A} / \mathrm{E} / \mathrm{A}$ ones

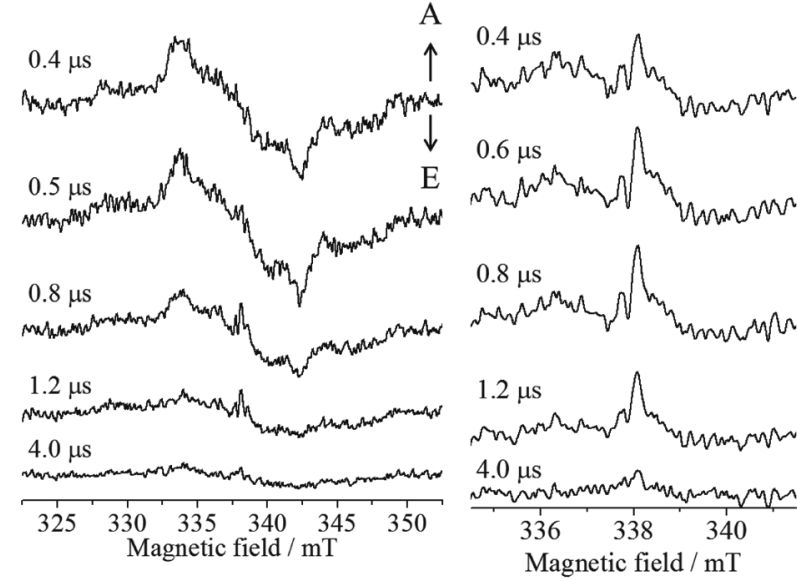

Fig. 2. (Left) Delay time dependence of the entire shape of the TREPR spectrum of the as-spun film of RR-P3HT:PC ${ }_{60} \mathrm{BM}=2: 8$ after the $532 \mathrm{~nm}$ laser excitations at $T=77 \mathrm{~K}$. (Right) Zoom-in views of the center field positions ( $338 \mathrm{mT}$ ) of the TREPR spectra obtained after the baseline corrections by the contributions from the excited triplet state of ${ }^{3} \mathrm{PC}_{60} \mathrm{BM}^{*}$.

indicates that both of short-range and long-range CS states are generated in highly disordered morphologies. The narrow EPR signal has been changed from the $\mathrm{A} / \mathrm{E} / \mathrm{A}$ to the weak absorptive signal at the later delay times by the spin lattice relaxation to generate the thermally equilibrium spin-state distribution. On the other hand, the broad $\mathrm{A} / \mathrm{E} / \mathrm{A} / \mathrm{E}$ pattern is quickly deactivated by the charge-recombination (CR).

As for the RR-P3HT:PC ${ }_{60} \mathrm{BM}=8: 2$ blend, the TREPR spectra were explained by sums of $\mathrm{A} / \mathrm{E} / \mathrm{A} / \mathrm{E}$ spectrum contributions and sharp emissive spectra at the field corresponding to $g=$ 2.002. Since the sharp emissive peak has been obtained by the $532 \mathrm{~nm}$ laser irradiation for the pristine RR-P3HT film at $77 \mathrm{~K}$, this spectrum contribution has been subtracted from the TREPR spectra in the 8:2 blend system as the photo-carriers generated in the P3HT-rich domains. The subtracted TREPR spectra shown in Fig. $3 \mathrm{~b}$ are thus attributable to the photoinduced CS states generated at the RR-P3HT:PC ${ }_{60} \mathrm{BM}$ domain interfaces. In the RR-P3HT:PC ${ }_{60} \mathrm{BM}=2: 8$ blend system (Fig. 3a), the broad $\mathrm{A} / \mathrm{E} / \mathrm{A} / \mathrm{E}$ polarization is predominant at the initial delay time of $0.4 \mu \mathrm{s}$. The $\mathrm{A} / \mathrm{E} / \mathrm{A} / \mathrm{E}$ spectrum-width is significantly sharper in the RR-P3HT:PC ${ }_{60} \mathrm{BM}=8: 2$ blend film as shown in Fig. 3b. This significant line-shape modulation by the polymer content is evidently associated with the crystallinity of the P3HT domain as shown in RR-P3HT:PC ${ }_{60} \mathrm{BM}=8: 2$ blend film as shown in Fig. 3b. This significant line-shape modulation by 
$\begin{array}{ll}\text { a) RR-P3HT:PCBM }=2: 8 & \text { b) RR-P3HT:PCBM }=8: 2\end{array}$

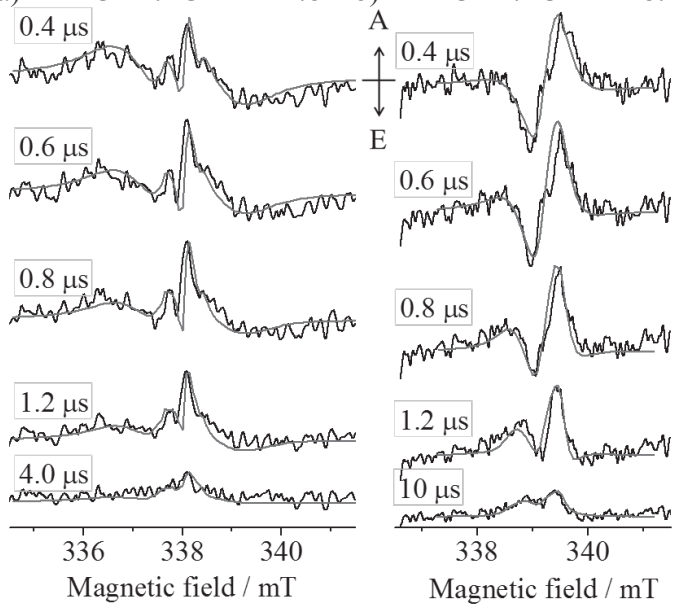

Fig. 3. Delay time dependences of the TREPR spectra of the interfacial photoinduced CS states in the as-spun films of (a) RR-P3HT:PC ${ }_{60} \mathrm{BM}=2: 8$ and of (b) RR-P3HT:PC ${ }_{60} \mathrm{BM}=8: 2$ after the $532 \mathrm{~nm}$ laser excitations at $T=77 \mathrm{~K}$. Computer simulations of the time-dependent EPR signals are superimposed on the experimental ones.

the polymer content is evidently associated with the crystallinity of the P3HT domain as shown in Fig. 1; the crystalline phase by the self-organized polymer molecules will cause the long-range $\mathrm{P}_{3} \mathrm{HT}^{+}: \mathrm{PC}_{60} \mathrm{BM}^{-} \quad \mathrm{CS}$ state resulting in significantly weakened spin-spin dipolar-coupling $(D)$ between the two spins. Additionally, the sharper CS-state survives more than $10 \mu \mathrm{s}$ in Fig. 3b, showing that the crystallinity contributes to the efficient charge dissociation, which is again consistent with the above distant CS state generations. The molecular geometries of the CS states can been obtained by utilizing the SCRP model, since the spin-polarized EPR spectra are affected by the $D$ coupling, the g-tensor orientations and the hyperfine anisotropies in the interfacial CS states, as reported in the previous study [6]. To clarify the electron-hole dissociation mechanism and the electronic property of the photoinduced CS states, the nanostructures and the $2 J$ were obtained by the analyses of the TREPR data (fitted curves in Fig. 3) on the basis of the stochastic-Liouville equation (SLE) by which the time-dependent quantum mechanical phenomena in the density matrix $(\rho)$ of the singlet-triplet system are solved in the rotating frame on the basis spin-functions of $\left.\left.\left|\mathrm{T}_{+}\right\rangle=|\alpha \alpha\rangle, \mathrm{IT}_{0}\right\rangle=|\alpha \beta+\beta \alpha\rangle / \sqrt{2}, \mathrm{IT} \mathrm{T}_{-}\right\rangle=|\beta \beta\rangle$ for the triplet CS state, and of IS $\rangle=\mid \alpha \beta-\beta \alpha$ $>/ \sqrt{ } 2$ for the singlet CS state $[6,8,9]$. The effects of the spin relaxations (the spin lattice relaxation time of $T_{1}$, the transverse relaxation times $T_{2}{ }^{*}$ and a) RR-P3HT:PCBM = 2:8
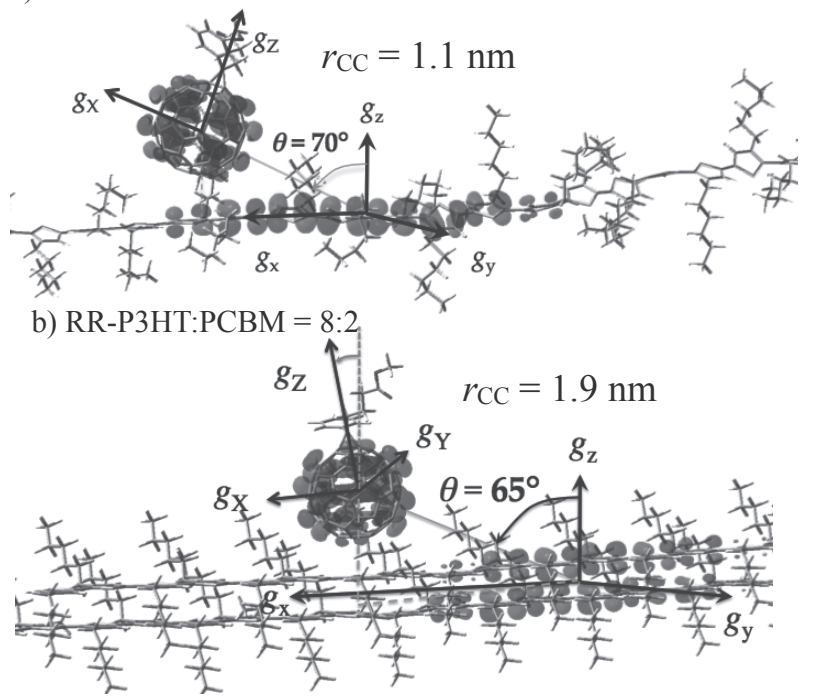

Fig. 4. Geometries of the photoinduced interfacial CS states to reproduce the spin-polarized TREPR spectra in Fig. $3 a$ and $3 b$ for the as-spun films of RR-P3HT:PC ${ }_{60} \mathrm{BM}=2: 8$ in a) and $8: 2$ in b), respectively at $T=77 \mathrm{~K} . r_{\mathrm{CC}}$ is the distance between the two spins.

the relaxation time of $T_{23}$ by the S- $\mathrm{T}_{0}$ dephasing) [10] and the CR kinetics were taken into account in the SLE $[6,8]$. Theoretical details how one can compute and evaluate the time-evolutions of the transverse magnetizations have been reported in our previous studies [5,6,11].

Figure 4 shows the geometries of the interfacial CS states to reproduce the TREPR spectra in Fig. 3. From Fig. $3 \mathrm{a}, D=-2.2 \mathrm{mT}$ and $J=27 \mu \mathrm{T}$ were determined. Using the point dipole approximation, center-to-center separation $\left(r_{\mathrm{CC}}\right)$ between $\mathrm{P}_{3} \mathrm{HT}^{+}$. and $\mathrm{PC}_{60} \mathrm{BM}^{-}$is obtained to be $r_{\mathrm{CC}}=1.1 \mathrm{~nm}$ from $D=-2.2 \mathrm{mT}$. The polymer chains are highly disordered in this experimental condition due to the lack of the excition band around 500-610 nm in Fig. 1. Thus, it is expected that the interfacial electron-hole pairs are generated in the P3HT:PC ${ }_{60} \mathrm{BM}$ molecular complex as shown in Fig. 4a. The polar angle of $\theta=70^{\circ}$ (the direction of the inter-spin vector with respect to the $g_{z}$ principal axes in $\mathrm{P}_{3} \mathrm{HT}^{+}$) has been essential to reproduce the $\mathrm{A} / \mathrm{E} / \mathrm{A} / \mathrm{E}$ pattern in Fig. 3a together with the azimuthal angle $\phi=10^{\circ}$. This $\theta$ close to $90^{\circ}$ denotes that the hole-dissociation takes place in the polymer backbone after the photoinduced charge-transfer reaction, as shown in Fig. 4a. When the RR-P3HT content is higher, $D=-0.38$ $\mathrm{mT}$ and $J=7 \mu \mathrm{T}$ were determined with $\theta=65^{\circ}$ and $\phi=35^{\circ}$ to reproduce the narrower $\mathrm{A} / \mathrm{E} / \mathrm{A} / \mathrm{E}$ line-shapes in Fig. 3b. The substantially weaker $D$ coupling represents that the transient holes are 
highly separated $\left(r_{\mathrm{CC}}=1.9 \mathrm{~nm}\right.$ from $D=-0.38$ $\mathrm{mT}$ ) by generating the highly delocalized spin distribution as shown in Fig. 4b. The structure of $\mathrm{P}_{3} \mathrm{HT}^{+}$in the P3HT domain is based upon an electron diffraction analysis that has revealed a tilted packing structure $[12,13]$ with the short $\pi-\pi$ stacking of a $3.4 \AA$ inter-planer distance in the crystalline P3HT. This $\pi-\pi$ stacking is coincident with the UV-vis absorption spectrum (Fig. 1) in the 8:2 RR-P3HT:PC ${ }_{60} \mathrm{BM}$ film, showing dominant strong vibrational absorption bands from $500 \mathrm{~nm}$ to $610 \mathrm{~nm}$ which are characteristics to the crystalline phase by the RR-P3HT molecules [14]. The inter-chain delocalized hole distribution is quite consistent with the dimer polaron model [15] which explains the absorption spectra of the polarons reported in the crystalline RR-P3HT phase. Such a delocalized hole in Fig. $4 \mathrm{~b}$ is also well consistent with the weaker exchange coupling $(J=7 \mu \mathrm{T})$ than $27 \mu \mathrm{T}$ in Fig. $4 \mathrm{a}$; the delocalized hole distribution will substantially reduce the orbital overlap between $\mathrm{P}_{3} \mathrm{HT}^{+\cdot}$ and $\mathrm{PC}_{60} \mathrm{BM}^{-}$in Fig. $4 \mathrm{~b}$ to contribute to the retarded CR process which is identified by the longer-lived CS state observation more than 10 $\mu \mathrm{s}$ in Fig. 3b. In the previous study, $D=-0.63 \mathrm{mT}$ was reported with $\theta=50^{\circ}$ for the as-spun RR-P3HT:PC ${ }_{60} \mathrm{BM}=5: 5$ film [5]. Both of the present weaker dipolar coupling and the larger $\theta$ in Fig. $4 \mathrm{~b}$ than in the 5:5 system denote that the transient holes are more separated from $\mathrm{PC}_{60} \mathrm{BM}$ toward the polymer long-axis direction rather than to the inter-chain $\pi-\pi$ stacking direction. From the UV-vis absorption spectra in Fig. 1, the band intensity at $610 \mathrm{~nm}$ is comparable to the intensity at $500 \mathrm{~nm}$ in the RR-P3HT:PC ${ }_{60} \mathrm{BM}=$ $8: 2$ system, while the $610 \mathrm{~nm}$ band intensity is much smaller than the $500 \mathrm{~nm}$ band in the 5:5 system. This enhanced low-energy band intensity is explained by the stronger J-type exciton coupling, indicating that highly ordered sheet-structures in the aromatic planes are produced at the crystalline phase. Such enhanced planner P3HT structures will also amplify the intra-chain electronic coupling for the hole-dissociations rather than to the inter-chain stacking direction as shown in Fig. $4 \mathrm{~b}$, resulting in $\theta=65^{\circ}$ which is larger than $50^{\circ}$.

In summary, we have investigated the morphology effect on the nanostructure and the exchange coupling of the interfacial CS states in the RR-P3HT:PC ${ }_{60} \mathrm{BM}$ film. In the lower RR-P3HT content, the polymer crystalline phase is not formed by the strong interactions from the $\mathrm{PC}_{60} \mathrm{BM}$ molecules, resulting in the short-range $\mathrm{CS}$ states with the quick energy-wasting recombination. For the higher polymer content, it has been demonstrated that the $\mathrm{PC}_{60} \mathrm{BM}$ molecules bind to the crystalline polymer regions at the BHJs and that the hole-dissociations take place accompanying the delocalized hole distribution in Fig. 4b, resulting in the long-lived photocarrier generations. The above structural and electronic properties are essential keys to evaluations, designs, and developments of the highly efficient solar energy-conversion systems employing organic polymers in the OPV systems.

\section{References}

1. G. Yu, J. Gao, J. C. Hummelen, F. Wudl and A. J. Heeger, Science, 270 (1995) 1789.

2. I. Osaka, M. Saito, T. Koganezawa and K. Takimiya, Adv. Mater., 26 (2014) 331.

3. S. Schumann, R. Da Campo, B. Illy, A. C. Cruickshank, M. A. McLachlan, M. P. Ryan, D. J. Riley, D. W. McComb and T. S. Jones, J. Mater. Chem., 21 (2011) 2381.

4. Y. Kim, S. Cook, S. M. Tuladhar, S. A. Choulis, J. Nelson, J. R. Durrant, D. D. C. Bradley, M. Giles, I. Mcculloch, C. S. Ha and M. Ree, Nat. Mater, 5 (2006) 197.

5. T. Miura, M. Aikawa and Y. Kobori, J. Phys. Chem. Lett., 5 (2014) 30.

6. Y. Kobori, R. Noji and S. Tsuganezawa, J. Phys. Chem. C, 117 (2013) 1589.

7. L. Franco, A. Toffoletti, M. Ruzzi, L. Montanari, C. Carati, L. Bonoldi and R. Po', J. Phys. Chem. C, 117 (2013) 1554.

8. T. Miura, M. Aikawa and Y. Kobori, J. Phys. Chem. Lett. 5 (2014) 30.

9. G. Kothe, S. Weber, E. Ohmes, M. C. Thurnauer and J. R. Norris, J. Phys. Chem., 98 (1994) 2706.

10. T. Fukuju, H. Yashiro, K. Maeda, H. Murai and T. Azumi, J. Phys. Chem. A, 101 (1997) 7783.

11. Y. Kobori and T. Miura, J. Phys. Chem. Lett., 6 (2015) 113.

12. N. Kayunkid, S. Uttiya and M. Brinkmann, Macromolecules, 43 (2010) 4961.

13. S. Lilliu, T. Agostinelli, E. Pires, M. Hampton, J. Nelson and J. E. Macdonald, Macromolecules, 44 (2011) 2725.

14. G. Li, V. Shrotriya, J. S. Huang, Y. Yao, T. Moriarty, K. Emery and Y. Yang, Nat. Mater., 4 (2005) 864

15. X. M. Jiang, R. Österbacka, O. Korovyanko, C. P. An, B. Horovitz, R. A. J. Janssen and Z. V. Vardeny, Adv. Funct. Mater., 12 (2002) 587. 